\title{
Effect of normobaric hypoxic exercise on blood pressure in old individuals
}

\author{
Markus Hein $^{1} \cdot$ Kristine Chobanyan-Jürgens $^{2,3} \cdot$ Uwe Tegtbur $^{1} \cdot$ Stefan Engeli ${ }^{2} \cdot$ Jens Jordan $^{4} \cdot$ Sven Haufe $^{1}$ (i)
}

Received: 5 March 2020 / Accepted: 25 November 2020 / Published online: 23 December 2020

(c) The Author(s) 2020

\begin{abstract}
Purpose To test the hypothesis that the combination of endurance training and hypoxia leads to greater improvements in resting and exercise blood pressure in old sedentary individuals compared to endurance training only.

Methods We randomly assigned 29 old overweight participants (age: $62 \pm 6$ years, body mass index (BMI): $28.5 \pm 0.5 \mathrm{~kg} /$ $\mathrm{m}^{2}, 52 \% \mathrm{men}$ ) to single blind 8-week bicycle exercise in hypoxia (fraction of inspired oxygen $\left(\mathrm{F}_{\mathrm{I}} \mathrm{O}_{2}\right)=0.15$ ) or normoxia $\left(\mathrm{F}_{\mathrm{I}} \mathrm{O}_{2}=0.21\right)$. Brachial blood pressure was measured at rest, during maximal incremental exercise testing, and during a 30 min constant work rate test, at baseline and after the training period.

Results Work rate, heart rate and perceived exertion during training were similar in both groups, with lower oxygen saturation for participants exercising under hypoxia $(88.7 \pm 1.5$ vs. $96.2 \pm 1.2 \%, t(27)=-13.04, p<0.001,|g|=4.85)$. Office blood pressure and blood pressure during incremental exercise tests did not change significantly in either group after the training program. Systolic blood pressure during the constant work rate test was reduced after training in hypoxia $(160 \pm 18$ vs. $151 \pm 14 \mathrm{mmHg}, t(13)=2.44 p<0.05, \mid d \mathrm{l}=0.55)$ but not normoxia $(154 \pm 22 \mathrm{vs} .150 \pm 16 \mathrm{mmHg}, t(14)=0.75, p=0.46$, $|d|=0.18)$ with no difference between groups over time $\left(F=0.08, p=0.77, \eta^{2}=0.01\right)$.

Conclusion In old individuals hypoxia in addition to exercise does not have superior effects on office or exercise blood pressure compared to training in normoxia.
\end{abstract}

Trial registration number ClinicalTrials.gov No. NCT02196623 (registered 22 July 2014).

Keywords Hypoxia $\cdot$ Exercise $\cdot$ Blood pressure $\cdot$ Elderly

\section{Abbreviations}

ANCOVA Analysis of covariance

BMI Body mass index

$\mathrm{F}_{\mathrm{I}} \mathrm{O}_{2} \quad$ Fraction of inspired oxygen

HIF- $1 \alpha \quad$ Hypoxia inducible factor $1 \alpha$

Communicated by Massimo Pagani.

Sven Haufe

Haufe.sven@mh-hannover.de

1 Institute of Sports Medicine, Hannover Medical School, Carl-Neuberg-Str. 1, 30625 Hannover, Germany

2 Institute of Clinical Pharmacology, Hannover Medical School, Hannover, Germany

3 Department of Clinical Pharmacology and Pharmacoepidemiology, Heidelberg University Hospital, Heidelberg, Germany

4 Institute of Aerospace Medicine, German Aerospace Center and University of Cologne, Cologne, Germany
HOMA-IR Homeostasis model assessment insulin resistance index

RPE Rating of perceived exertion

$\mathrm{SaO}_{2} \quad$ Arterial oxygen saturation

SD Standard deviation

VEGF Vascular endothelial growth factor

$\mathrm{VO}_{2 \text { peak }} \quad$ Peak oxygen consumption

\section{Introduction}

Hypertension is a major risk factor for death (Whelton et al. 2017) and predicts disability-adjusted life years (Lim et al. 2012; Whelton et al. 2017). While aerobic capacity decreases, systolic blood pressure progressively increases (Benetos et al. 2002) with advancing age. Moreover, in old individuals, elevated blood pressure is associated with substantially higher absolute cardiovascular risk than in young individuals (Rapsomaniki et al. 2014). 
Physical activity improves blood pressure control (Cornelissen and Fagard 2005) in patients with arterial hypertension (Somers et al. 1991) and is equally effective in lowering systolic blood pressure as commonly prescribed antihypertensive medications (e.g. angiotensin-converting enzyme inhibitors, angiotensin-2 receptor antagonists, $\beta$-adrenoceptor antagonists, calcium channel blockers and diuretics) (Naci et al. 2019). However, endurance training may be limited by orthopedic comorbidities in old individuals. Training strategies that reduce the strain on the locomotor system, such as hypoxic exercise, may be of interest to practitioners working with this population. Thereby, less stress might be imposed on the musculoskeletal system while still realizing the same or even greater cardiovascular benefits of exercising (Haufe et al. 2008; Pramsohler et al. 2017).

The current data concerning the blood pressure response to hypoxia are contradictory. Intermittent hypoxia comprising intervals of breathing normoxic and hypoxic air without physical exercise has been previously tested in treating patients with arterial hypertension (Serebrovskaya et al. 2008). On the other hand, hypoxia may play a role in the pathogenesis of arterial hypertension (Kayser and Verges 2013) through repeated fluctuations in intrathoracic pressure caused by increased activity of the chemoreceptor-mediated sympathetic nervous system, resulting in increased heart rate, cardiac output and peripheral resistance (Malfatto, Ochoa and Parati 2009).

Breathing hypoxic air engages similar metabolic pathways as endurance exercise such as the hypoxia inducible factor 1 system (Ameln et al. 2005). Up regulation of hypoxia inducible factor $1 \alpha$ (HIF-1 $\alpha$ ) protein expression during exercise could further enhance activation of vascular endothelial growth factor (VEGF) (Ameln et al. 2005). VEGF plays a critical role in blood pressure control via nitric oxide synthase expression and nitric oxide activity (Facemire et al. 2009). Hypoxia-induced VEGF activation may also contribute to increased capillarization, which is also a typical response to endurance exercise (Hansen et al. 2010).

Previous studies have evaluated the combination of exercise in hypoxia for several health-related outcomes (González-Muniesa et al. 2015), yet, data from randomized controlled trials on blood pressure changes following hypoxic exercise are sparse. We tested the hypothesis that the combination of exercise and hypoxia lead to greater improvements in resting and exercise blood pressure compared to normoxic endurance exercise in old sedentary individuals.

\section{Methods}

This is a secondary analysis of a study testing the effects of hypoxic training on changes in whole body insulin sensitivity and oxidative metabolism as primary outcome (Chobanyan-Jürgens et al. 2019) (ClinicalTrials. gov Identifier: NCT02196623). We included women and men aged 55-75 years who were weight stable during the last 6 months ( $\pm 2 \%$ body weight), had a body mass index between 20 and $35 \mathrm{~kg} / \mathrm{m}^{2}$, and a homeostasis model assessment insulin resistance index (HOMA-IR) at screening between 2.0 and 4.0 units. Sixty-eight individuals were screened for the study of which 29 met the inclusion criteria and were randomized to hypoxic or normoxic training.

Detailed medical history, examination, 12-lead electrocardiogram, and blood sampling for routine laboratory tests assessed preexisting diseases. Exclusion criteria were more than $1 \mathrm{~h}$ of scheduled exercise training per week; known diagnosis of type 2 diabetes or $\mathrm{HbA} 1 \mathrm{c}>6.5 \%$ (>48 mmol/mol); smoking $>20$ cigarette/day; known alcohol or drug abuse; acute or chronic infections; increased bleeding risk by history or laboratory testing; any contraindication (orthopedic, cardiopulmonary etc.) to perform exercise training. The institutional review board of Hannover Medical School approved the study, and we obtained written informed consent before participants' enrollment.

The training program was reported in detail previously (Chobanyan-Jürgens et al. 2019). In brief, participants completed an 8-week supervised endurance training on a stationary bicycle ergometer (Optibike 50, ergoline, Bitz, Germany) in a normobaric hypoxic chamber, with ambient conditions (hypoxic $\left[\mathrm{F}_{\mathrm{I}} \mathrm{O}_{2}=0.15\right.$, corresponding to $2750 \mathrm{~m}$ altitude] or normoxic $\left.\left[\mathrm{F}_{\mathrm{I}} \mathrm{O}_{2}=0.21\right]\right)$ in a single-blinded fashion. Participants trained thrice weekly at similar relative exercise intensities in both intervention groups (heart rate corresponding to $60 \%$ of pre-training peak oxygen consumption $\left(V_{2 \text { peak }}\right)$ ) for $30 \mathrm{~min}$. To compensate for training-induced effects we increased exercise intensity to $70 \%$ of pre-training $V \mathrm{O}_{2 \text { peak }}$ and exercise duration to $40 \mathrm{~min}$ after the first 4 weeks). Heart rate and blood oxygen saturation (Rad-5, Masimo Corporation, Irvine, USA) were measured every ten minutes during training and rating of perceived exertion (RPE) (Borg scale) was documented at the end of every session.

Before (baseline) and after the training program (post), participants reported to the laboratory twice. On the first day participants rested on a chair in a quiet room for five minutes, before office blood pressure and heart rate (CARESCAPE V100, GE Healthcare, Boston, USA) were measured three times in one-minute intervals. Results are given as average of the three measurements. Afterwards, 
anthropometric data and body composition (BodPod, Life Measurement, Inc., Concord, USA) were determined. Finally participants performed a graded exercise test on a bicycle ergometer (ergometrics 900, ergoline, Bitz, Germany) starting at a work rate of $50 \mathrm{~W}$ increasing by $10 \mathrm{~W}$ every minute until volitional exhaustion. Throughout the test, we recorded heart rate using a 12-channel-electrocardiogram (CardioSoft, GE Healthcare, Boston, USA) and respiratory gas exchange breath-by-breath using indirect calorimetry (Masterscreen CPX, Becton Dickinson, Franklin Lakes, USA). Every three minutes, we measured blood pressure and obtained small blood samples from the hyperaemized earlobe to measure blood lactate concentrations (Biosen S-Line, EKF-diagnostic GmbH, Barleben, Germany). We determined $V \mathrm{O}_{\text {2peak }}$ and maximal heart rate as the highest $30 \mathrm{~s}$ average during the exercise test.

With at least two days rest in between, participants performed a submaximal exercise test at a work rate corresponding to $60 \%$ of their baseline $V \mathrm{O}_{2 \text { peak }}$ for $30 \mathrm{~min}$. We recorded heart rate and respiratory gas exchange as described above and blood pressure and blood lactate concentrations every five minutes throughout the test. Average heart rate, blood lactate concentration and respiratory exchange ratio were calculated as mean \pm standard deviation (SD) from minute 5 to 30. At the end of the test participants reported their RPE. After the training program, the submaximal test was performed at the same absolute work rate as at baseline and at least two days before the graded exercise test.

\section{Statistical analysis}

We conducted the statistical analysis in the intention-totreat population, defined as all randomized individuals who participated in at least one training session. Missing values were replaced by baseline values. As sensitivity analysis, we also performed a per-protocol analysis (only participants with pre- and post-intervention data and at least 23 out of 24 scheduled training sessions). Differences between groups at baseline were analyzed with an unpaired student's $t$ test. Within-group differences in blood pressures changes from pre- to post-training for the intervention and control group were calculated with student's t test for paired samples. Mean differences between groups over time were analyzed with an analysis of covariance (ANCOVA), adjusted for sex, age and the baseline value as covariates. Effect sizes for differences between groups were calculated using Hedges'g $(|g|)$ and for differences within groups (baseline-post) with Cohen's $d(|d|)$. For the ANCOVA, Eta-squared $\left(\eta^{2}\right)$ was used to calculate effect sizes between groups over time. Univariate associations between parameters were tested using Spearman's correlation coefficient.

Values are presented as mean \pm standard deviation (SD) unless otherwise stated. $p<0.05$ was considered to be statistically significant. All analyses were carried out with the SPSS software package for Windows ${ }^{\circledR}$ (Version 24, IBM Corp., Armonk, NY, USA).

\section{Results}

Twelve participants in the hypoxia group and thirteen participants in the normoxia group completed the training protocol. Two participants dropped out prior to completing the post-training tests in each group due to study unrelated events. For all outcomes assessed no further values had to be replaced post intervention, except for one individual in the normoxia group who did not perform the constant work rate test after the intervention and missing values were therefore replaced by the respective baseline values.

Demographic and physiological baseline characteristics for both treatment groups are given in Table 1. Average training work rate was $72 \pm 23 \mathrm{~W}$ in the hypoxia group and $83 \pm 33 \mathrm{~W}$ in the normoxia group $(t(27)=-1.00, p=0.33$, $|g|=0.37)$. Both groups trained at similar absolute heart rates (hypoxia: $114 \pm 14 \mathrm{bpm}$, normoxia: $115 \pm 10 \mathrm{bpm}$, $(t(27)=-0.89, p=0.91,|g|=0.04)$ and similar percentage of their maximal heart rate (hypoxia: $76 \pm 6 \%$, normoxia: $74 \pm 5 \%(t(27)=-0.12, p=0.30,|g|=0.40)$. Arterial oxygen saturation during training was significantly lower in the hypoxia $(89 \pm 1.5 \%)$ compared to the normoxia group $(96 \pm 1.5 \%)(t(27)=-13.04, p<0.001,|g|=4.85)$. Exercise performance parameters for the initial session of each week (training progression) are given in Table 2. Of those participants completing the 8-week intervention $(n=25)$ seventeen performed 24 out of 24 scheduled exercise training sessions (68\%) and five performed 23 out of 24 scheduled sessions (24\%). One individual in the normoxia and one in the hypoxia group performed 15 out of 24 exercise sessions (8\%).

After the training program body weight and body fat mass (hypoxia group: $-0.5 \pm 2.3 \mathrm{~kg}$; normoxia group:

Table 1 Demographic and physiological baseline characteristics

\begin{tabular}{|c|c|c|c|c|}
\hline & Hypoxia group & Normoxia group & $p$ & $|g|$ \\
\hline Age, years & $60.4 \pm 5.0$ & $63.8 \pm 5.8$ & 0.10 & 0.63 \\
\hline Body weight, kg & $86.7 \pm 10.3$ & $88.4 \pm 14.5$ & 0.72 & 0.13 \\
\hline BMI, $\mathrm{kg}^{*} \mathrm{~m}^{-2}$ & $28.6 \pm 3.0$ & $28.4 \pm 1.9$ & 0.71 & 0.14 \\
\hline RR sys, $\mathrm{mmHg}$ & $129.1 \pm 9.3$ & $133.9 \pm 13.5$ & 0.27 & 0.42 \\
\hline $\mathrm{RR}$ dia, $\mathrm{mmHg}$ & $81.6 \pm 8.5$ & $79.2 \pm 10.3$ & 0.49 & 0.26 \\
\hline $\begin{array}{l}\text { Resting heart rate, } \\
\text { bpm }\end{array}$ & $68 \pm 7$ & $66 \pm 9$ & 0.63 & 0.18 \\
\hline
\end{tabular}

Data are mean \pm SD. No significant differences between groups as analyzed with Student's $t$ test for unpaired samples. $|g|$ : Hedges' $g$

$B M I$ body mass index, $R R$ sys systolic blood pressure, $R R$ dia diastolic blood pressure 


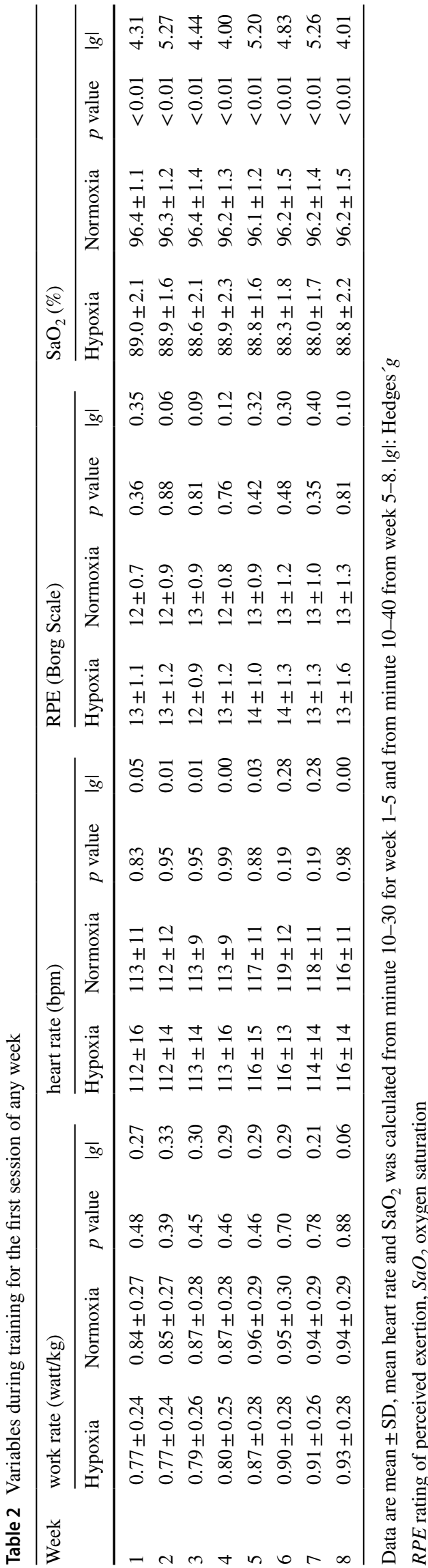

$-0.7 \pm 1.6 \mathrm{~kg}$; between-groups: $t(27)=0.26, p=0.80$, $|g|=0.10)$ did not change significantly with training irrespective of oxygen conditions. The improvement of $V \mathrm{O}_{2 \text { peak }}$ was only statistically significant in the normoxia training group (Table 3) with no differences between groups.

Eight weeks of endurance training in hypoxic and normoxic conditions did not significantly change systolic (hypoxia baseline: $129 \pm 9 \mathrm{mmHg}$; post: $127 \pm 8 \mathrm{mmHg}$, $t(13)=1.42, p=0.18,|d|=0.21$; normoxia baseline: $134 \pm 14 \mathrm{mmHg}$; post: $132 \pm 12 \mathrm{mmHg}, t(14)=1.08 p=0.30$, $|d|=0.17$ ) or diastolic (hypoxia baseline: $82 \pm 9 \mathrm{mmHg}$; post: $81 \pm 7 \mathrm{mmHg}, t(13)=0.19, p=0.85,|d|=0.05$; normoxia baseline: $79 \pm 10 \mathrm{mmHg}$; post: $78 \pm 11 \mathrm{mmHg}$, $t(14)=0.50 p=0.62,|d|=0.08)$ office blood pressure (see also Fig. 1). A per-protocol analysis $(n=23)$ showed similar results for office systolic (hypoxia baseline: $128 \pm 9 \mathrm{mmHg}$; post: $126 \pm 8 \mathrm{mmHg}, t(10)=1.12, p=0.29,|d|=0.20$; normoxia baseline: $134 \pm 12 \mathrm{mmHg}$; post: $132 \pm 11 \mathrm{mmHg}$, $t(11)=0.73 p=0.48,|d|=0.16)$ and diastolic blood pressure (hypoxia baseline: $81 \pm 9 \mathrm{mmHg}$; post: $81 \pm 7 \mathrm{mmHg}$, $t(10)=0.69, p=0.35,|d|=0.18$; normoxia baseline: $81 \pm 9 \mathrm{mmHg}$; post: $80 \pm 10 \mathrm{mmHg}, t(11)=0.26 p=0.80$, $|d|=0.05)$.

During the constant work rate exercise test, systolic blood pressure was significantly reduced after training within the hypoxia group, $(160 \pm 18$ to $151 \pm 14 \mathrm{mmHg}$, $t(13)=2.44 p<0.05,|d|=0.55)$, but not the normoxia group $(154 \pm 22 \mathrm{mmHg}$ to $150 \pm 16 \mathrm{mmHg}, t(14)=0.75$, $p=0.46,|d|=0.18$ ), (see also Fig. 2), with no differences between groups over time $\left(F=0.08, p=0.77, \eta^{2}=0.01\right)$. Both groups showed reduced average heart rate (hypoxia baseline: $116 \pm 21 \mathrm{bpm}$; post: $110 \pm 20 \mathrm{bpm}, t(13)=2.39$, $p<0.05,|d|=0.29$; normoxia baseline: $114 \pm 14 \mathrm{bpm}$; post: $107 \pm 13 \mathrm{bpm}, t(14)=2.40 p<0.05,|d|=0.48)$, blood lactate concentration (hypoxia baseline: $2.5 \pm 1.1 \mathrm{mM}$; post: $1.9 \pm 1.2 \mathrm{mM}, t(13)=3.48, p<0.05,|d|=0.52$; normoxia baseline: $2.2 \pm 0.8 \mathrm{mM}$; post: $1.7 \pm 0.7 \mathrm{mM}, t(14)=2.97$ $p<0.05,|d|=0.65$ ) (see also Fig. 2 for data at single time points), respiratory exchange ratio (hypoxia baseline: $0.97 \pm 0.05$; post: $0.94 \pm 0.06, t(13)=2.48, p<0.05$, $|d|=0.59$; normoxia baseline: $0.95 \pm 0.04$; post: $0.92 \pm 0.04$, $t(14)=2.18 p<0.05,|d|=0.63)$ and perceived exertion (hypoxia baseline: $13.5 \pm 1.3$; post: $12.8 \pm 1.6, t(13)=2.68$, $p<0.05,|d|=0.48$; normoxia baseline: $12.8 \pm 0.7$; post: $11.7 \pm 1.8, t(14)=2.87 p<0.05,|d|=0.79)$ during constant work rate tests after the training program.

We did not observe significant correlations between training compliance (percentage of completed exercise sessions) and intervention-induced changes in blood pressure at rest (systolic BP: Rho: 0.01, $p=0.98$; diastolic BP: Rho: 0.08, $\mathrm{p}=0.67$ ) or during constant submaximal exercise (systolic BP: Rho: 0.17, $p=0.38$; diastolic BP: Rho: 0.07, $p=0.72$ ). 
Table 3 Performance variables during graded exercise test

\begin{tabular}{|c|c|c|c|c|c|c|c|c|}
\hline & \multicolumn{3}{|c|}{ Hypoxia group } & \multicolumn{3}{|c|}{ Normoxia group } & \multirow{2}{*}{$\begin{array}{l}\text { Group } \mathrm{x} \text { time } \\
p \text { value }\end{array}$} & \multirow[b]{2}{*}{$\eta^{2}$} \\
\hline & Baseline & Post & $|d|$ & Baseline & Post & $|d|$ & & \\
\hline Maximal power, $\mathrm{W}$ & $154 \pm 41$ & $167 \pm 46^{*}$ & 0.30 & $161 \pm 55$ & $169 \pm 56^{*}$ & 0.14 & 0.29 & 0.05 \\
\hline Relative maximal power, W/kg & $1.8 \pm 0.4$ & $2.0 \pm 0.5^{*}$ & 0.44 & $1.8 \pm 0.4$ & $1.9 \pm 0.5^{*}$ & 0.22 & 0.13 & 0.09 \\
\hline $\mathrm{VO}_{2}$ peak, $\mathrm{ml} / \mathrm{min}$ & $2090 \pm 501$ & $2232 \pm 587$ & 0.26 & $2090 \pm 665$ & $2210 \pm 636^{*}$ & 0.18 & 0.60 & 0.01 \\
\hline
\end{tabular}

Data are mean $\pm \mathrm{SD}, * p<0.05$

$|d|$ Cohen's $d, \eta^{2}$ eta-squared, $V O_{2}$ oxygen uptake
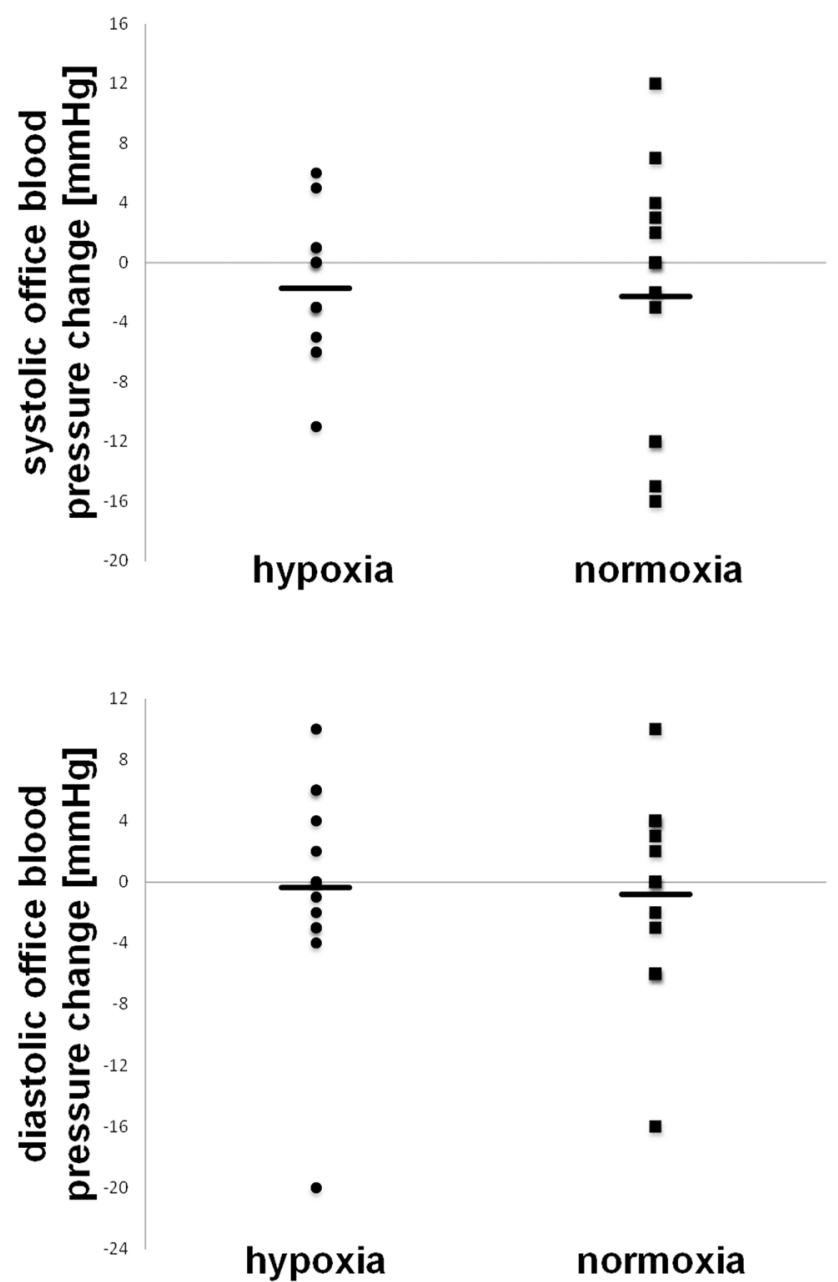

Fig. 1 Individual and mean (solid line) changes in office blood pressure of participants randomized to exercise in normoxia or hypoxia after 8 weeks of training compared to baseline. No significant differences were detected within groups or between groups over time

\section{Discussion}

The main finding of our study is that 8 weeks hypoxic endurance training had no major and consistent effect beyond that of exercise in normoxia on resting and exercise blood pressure in old sedentary individuals.

Age-induced blood pressure elevation is thought to be mainly due to atherosclerotic changes, stiffening of large arteries, renal dysfunction, and arterial baroreflex impairment (Benetos et al. 2002). Many of the changes occurring with advanced age are associated with a decrease in physical activity and cardiorespiratory fitness (Milanović et al. 2013). Physical training can be a potential treatment strategy to reduce blood pressure, but its effects are controversial and may vary between individuals (Cornelissen and Fagard 2005; Somers et al. 1991).

Beneficial effects of regular hypoxic training on blood pressure regulation have been observed in some studies. Three weeks of moderate sports activities at natural $1700 \mathrm{~m}$ altitude showed a significant decrease in systolic and diastolic blood pressure in individuals with metabolic syndrome (Schobersberger et al. 2003). Kong et al. and Morishima et al. reported a $10 \mathrm{mmHg}$ and $7 \mathrm{mmHg}$ decrease in systolic blood pressure after 4 weeks exercise training at 16.4-14.5\% $\mathrm{F}_{\mathrm{I}} \mathrm{O}_{2}$ (Kong et al. 2014; Morishima et al. 2015), whereas González-Muniesa et al. only observed a reduction in diastolic blood pressure after an 8 -week strength and endurance protocol in normobaric hypoxia simulating $2000-3350 \mathrm{~m}$ altitude (González-Muniesa et al. 2015). We could not confirm these results in our cohort. Most studies reported improved body composition after the training period which is a crucial factor in decreasing blood pressure by exercise (Stewart et al. 2005). In our study individuals' weight remained stable, which might partly explain different outcomes to other studies in which weight loss might also be a result of the additional dietary advice given to participants (González-Muniesa et al. 2015; Kong et al. 2014; Schobersberger et al. 2003). Further reasons for the lack of favorable effects of our tested hypoxic training program might include insufficient duration, frequency and severity of the hypoxic episodes as these seems to be critical factors in determining whether intermittent hypoxia is beneficial (Manukhina et al. 2006; Serebrovskaya and Xi 2016). Moreover, there is some evidence that old individuals might be resistant to exerciseinduced blood pressure reduction (Stewart et al. 2005) and the change of body composition is a decisive factor in this regard (Kong et al. 2014; Stewart et al. 2005). 

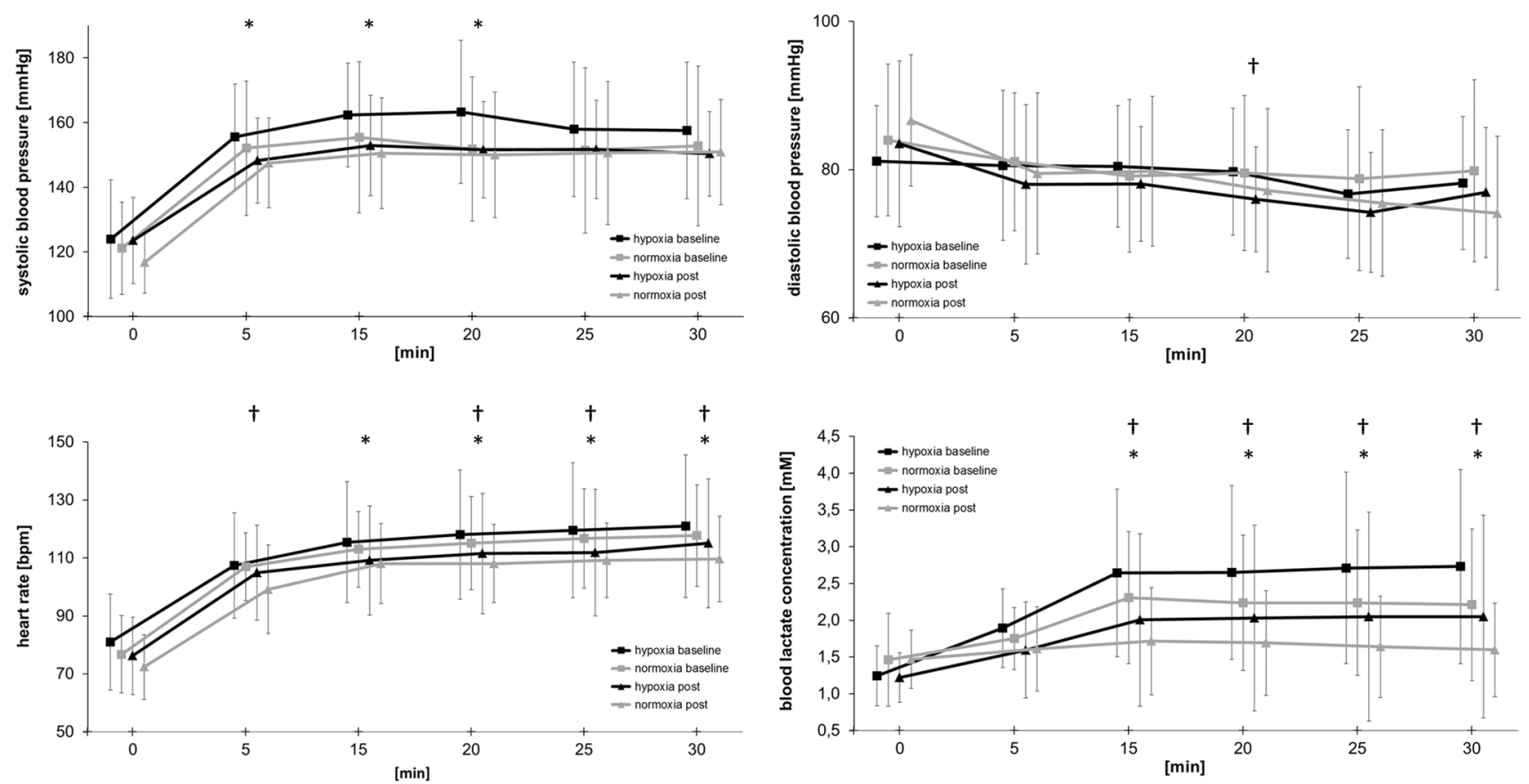

Fig. 2 Blood pressure, heart rate, and blood lactate concentration during constant work rate exercise test $(60 \%$ of baseline peak oxygen uptake) on a bicycle ergometer at baseline and after 8 weeks. Data

are mean \pm SD. ${ }^{*} p<0.05$ between baseline and post in the hypoxia group. $\dagger \mathbf{p}<0.05$ between baseline and post in the normoxia group. No significant differences were detected between groups over time

Yet, our results are in line with some other studies where hypoxic exercise did not have significant positive effects on arterial blood pressure Fu et al. 2007: (systolic: $\pm 0 \mathrm{mmHg}$, diastolic: $+1 \mathrm{mmHg}$ ), Gatterer et al. 2015 (systolic: $-4 \mathrm{mmHg}$, diastolic: $-2 \mathrm{mmHg}$ ), Pramsohler et al. 2017 (hypoxia: systolic: $\pm 0 \mathrm{mmHg}$, diastolic: $\pm 0 \mathrm{mmHg}$ ), Wang et al. 2007 (hypoxia: systolic: $+3 \mathrm{mmHg}$, diastolic: $+2 \mathrm{mmHg}$ ), and Wiesner et al. 2009 (systolic: $-2 \mathrm{mmHg}$, diastolic: $-3 \mathrm{mmHg}$ ). Notably, endurance training does not always change blood pressure neither in normotensive nor in hypertensive individuals (Cornelissen and Smart 2013).

Hypoxic exercise training has been shown to decrease arterial stiffness (Nishiwaki et al. 2010), metabolic risk factors like body fat (Haufe et al. 2008; Wiesner et al. 2009) and insulin resistance (Haufe et al. 2008; Mackenzie et al. 2011; Morishima et al. 2013), which all have been implicated in the pathogenesis of hypertension. As an acute effect, endurance exercise as wells as hypoxia result in vasodilation (Nishiwaki et al. 2010) and decreased blood pressure (Somers et al. 1991; Parati et al. 2015). These effects are induced, amongst others, by increased HIF- $1 \alpha$ protein expression, triggered by increased oxygen consumption or lowered tissue oxygen tension (Ameln et al. 2005). HIF-1 $\alpha$ elicits VEGF activation (Ameln et al. 2005), which acutely affects blood pressure control via nitric oxide synthase expression and nitric oxide activity

(Blitzer et al. 1996; Facemire et al. 2009). The activation of the HIF pathway appears to be stronger with endurance exercise performed under hypoxic conditions (Vogt et al. 2001). Therefore, combining these two stimuli might be a reasonable approach to attain synergistic benefits on acute and chronic blood pressure control (Casey et al. 2010; Beck et al. 2013; Pedralli et al. 2020).

Controversial to these positive effects, deleterious reactions may also occur during acute hypoxic exposure (Manukhina et al. 2006). Intermittent episodes of breathing hypoxic air have been used as a model for sleep apnea and caused hypertension in animal models (Fu et al. 2007; Manukhina et al. 2006). Stimulation of the peripheral chemoreceptors, which leads to an increase in sympathetic tone and a decrease in vagal activity, can cause an acute increase in blood pressure (Fu et al. 2007). However, these adverse effects seem to occur during severe and/or long lasting hypoxic exposure (Manukhina et al. 2006), not comparable to short-term exposure as applied in our study.

We did not observe improvements in cardiorespiratory fitness $\left(\mathrm{VO}_{2}\right.$ peak) after 8 weeks, which might indicate that our training program is unlikely to change other health-related outcomes. We chose this protocol as it reflects guideline recommendations (ACSM 1993) and is considered to positively affect cardiorespiratory fitness and blood pressure even with small sample sizes (Badenhop et al. 1983). Despite no change in $V \mathrm{O}_{2 \text { peak }}$, maximal power increased significantly 
in both study groups, which indicates a partially successful training protocol with regard to physical fitness.

While the observed systolic office blood pressure reduction in our study ( $2 \mathrm{mmHg}$ ) did not reach statistical significance in either group, the magnitude of pressure reduction is similar to those reported in previous meta-analysis (Cornelissen and Fagard 2005; Cornelissen and Smart 2013). Although the change was small, meta-analyses suggest that a $2 \mathrm{mmHg}$ systolic blood pressure decrease reduces the risk of major cardiovascular disease events by about $4 \%$, stroke by $5 \%$, and heart failure by $6 \%$ (Ettehad et al. 2016; Cornelissen and Fagard 2005). Hence, our training program showed potential to exert clinically relevant reductions in office blood pressure; however, normobaric hypoxia did not further improve these responses compared with exercise alone.

As no adverse effects or discomfort occurred during our training program, normobaric hypoxic training at simulated $2700 \mathrm{~m}$ altitude $\left(\mathrm{F}_{\mathrm{I}} \mathrm{O}_{2}=0.15 \%\right)$ appears to be feasible to apply in old individuals. A potential benefit of hypoxic training could be the performance of exercise at reduced work rate and thereby less stress on the locomotor system. This was reported by some studies (Haufe et al. 2008; Pramsohler et al. 2017) whereas others did not report such differences. We observed a $15 \%$ lower work rate at average for those exercising under normobaric hypoxia, which does not reach statistical significance. Nevertheless this data imply to further studying hypoxic training in larger, but also various target groups as an approach for attaining similar cardiovascular or metabolic benefits after exercise training, while performing at lower intense work rates.

Our study has strengths and limitations. Blood pressure changes were assessed at rest and during exercise in a population in which the risk for hypertension is elevated. Although continuous physical activity would be important for these individuals, this task is often limited by locomotor constriction, which may offer a potential for hypoxic training because of reduced work rate.

As a limitation, the prescription of exercise intensity based on pre-training $\% \mathrm{VO}_{2}$ max may have produced different training stimuli across participants, which may have led to a training stimulus not sufficient to induce changes in blood pressure in some participants. We used an intentionto-treat analysis with baseline observation carried forward for missing data as a conservative statistical method, as it avoids possible bias associated with loss of patients in a controlled trial. However, since this approach has also potential weaknesses, we conducted a per-protocol analysis (only cases with pre- and post-intervention data) in addition to our a priori defined analysis.

In conclusion, 8 weeks of hypoxic exercise in old women and men is a safe and feasible training method. However, it is not superior over normoxic training at the same relative exercise intensity. It needs to be further examined if longer durations of hypoxic episodes or higher training intensities have more beneficial effects.

Author contributions $\mathrm{SH}$, UT and JJ conceived and designed research. $\mathrm{MH}, \mathrm{KC}, \mathrm{SE}$ and $\mathrm{SH}$ conducted experiments. $\mathrm{MH}$ and $\mathrm{SH}$ analyzed data. MH wrote the manuscript. SE, JJ and SH revised the manuscript. All authors read and approved the manuscript.

Funding Open Access funding enabled and organized by Projekt DEAL.. This work was supported by a Grant from the German Research Foundation (Reference No. HA 7037/ 2-1) to SH.

Availability of data and material The datasets generated during and/or analyzed during the current study are available from the corresponding author on reasonable request.

\section{Compliance with ethical standards}

Conflict of interest The authors have nothing to disclose.

Ethics approval The institutional review board of Hannover Medical School approved the study.

Consent to participate Written informed consent was obtained from each participant before enrollment.

Consent for publication Written informed consent was obtained from each participant before enrollment.

Open Access This article is licensed under a Creative Commons Attribution 4.0 International License, which permits use, sharing, adaptation, distribution and reproduction in any medium or format, as long as you give appropriate credit to the original author(s) and the source, provide a link to the Creative Commons licence, and indicate if changes were made. The images or other third party material in this article are included in the article's Creative Commons licence, unless indicated otherwise in a credit line to the material. If material is not included in the article's Creative Commons licence and your intended use is not permitted by statutory regulation or exceeds the permitted use, you will need to obtain permission directly from the copyright holder. To view a copy of this licence, visit http://creativecommons.org/licenses/by/4.0/.

\section{References}

Ameln H, Gustafsson T, Sundberg CJ et al (2005) Physiological activation of hypoxia inducible factor-1 in human skeletal muscle. FASEB J 19:1009-1011. https://doi.org/10.1096/fj.04-2304fje

American College of Sports Medicine Position Stand (1993) Physical activity, physical fitness, and hypertension. Med Sci Sports Exer 25:i-x

Badenhop DT, Cleary PA, Schaal SF et al (1983) Physiological adjustments to higher- or lower-intensity exercise in elders. Med Sci Sports Exerc 15:496-502

Beck DT, Martin JS, Casey DP, Braith RW (2013) Exercise training improves endothelial function in resistance arteries of young prehypertensives. Exp Biol Med (Maywood) 238:433-441. https:// doi.org/10.1038/jhh.2013.109

Benetos A, Waeber B, Izzo J et al (2002) Influence of age, risk factors, and cardiovascular and renal disease on arterial stiffness: 
clinical applications. Am J Hypertens 15:1101-1108. https://doi. org/10.1016/s0895-7061(02)03029-7

Blitzer ML, Lee SD, Creager MA (1996) Endothelium-derived nitric oxide mediates hypoxic vasodilation of resistance vessels in humans. Am J Physiol 271:H1182-H1185. https://doi. org/10.1152/ajpheart.1996.271.3.H1182

Casey DP, Madery BD, Curry TB et al (2010) Nitric oxide contributes to the augmented vasodilatation during hypoxic exercise. $\mathrm{J}$ Physiol (Lond) 588:373-385. https://doi.org/10.1152/japplphysi ol.00487.2006

Chobanyan-Jürgens K, Scheibe RJ, Potthast AB et al (2019) Influences of hypoxia exercise on whole body insulin sensitivity and oxidative metabolism in older individuals. J Clin Endocrinol Metab. https://doi.org/10.1210/jc.2019-00411

Cornelissen VA, Fagard RH (2005) Effects of endurance training on blood pressure, blood pressure-regulating mechanisms, and cardiovascular risk factors. Hypertension 46:667-675. https:// doi.org/10.3109/07853899109148065

Cornelissen VA, Smart NA (2013) Exercise training for blood pressure: a systematic review and meta-analysis. J Am Heart Assoc 2:103. https://doi.org/10.1136/bmj.316.7139.1236

Ettehad D, Emdin CA, Kiran A et al (2016) Blood pressure lowering for prevention of cardiovascular disease and death: a systematic review and meta-analysis. Lancet 387:957-967. https://doi. org/10.1016/S0140-6736(15)01225-8

Facemire CS, Nixon AB, Griffiths R et al (2009) Vascular endothelial growth factor receptor 2 controls blood pressure by regulating nitric oxide synthase expression. Hypertension 54:652-658. https://doi.org/10.1016/B978-012088488-9.50026-7

Fu Q, Townsend NE, Shiller SM et al (2007) Intermittent hypobaric hypoxia exposure does not cause sustained alterations in autonomic control of blood pressure in young athletes. Am J Physiol Regul Integr Comp Physiol 292:R1977-R1984. https ://doi.org/10.1152/ajpregu.00622.2006

Gatterer H, Haacke S, Burtscher M et al (2015) Normobaric intermittent hypoxia over 8 months does not reduce body weight and metabolic risk factors-a randomized, single blind, placebocontrolled study in normobaric hypoxia and normobaric sham hypoxia. Obes Facts 8:200-209. https://doi.org/10.1159/00043 1157

González-Muniesa P, Lopez-Pascual A, de Andrés J et al (2015) Impact of intermittent hypoxia and exercise on blood pressure and metabolic features from obese subjects suffering sleep apnea-hypopnea syndrome. J Physiol Biochem 71:589-599. https://doi.org/10.1056/NEJMoa1008234

Hansen AH, Nielsen JJ, Saltin B, Hellsten Y (2010) Exercise training normalizes skeletal muscle vascular endothelial growth factor levels in patients with essential hypertension. J Hypertens 28:1176-1185. https://doi.org/10.1097/HJH.0b013e3283379120

Haufe S, Wiesner S, Engeli S et al (2008) Influences of normobaric hypoxia training on metabolic risk markers in human subjects. Med Sci Sports Exerc 40:1939-1944. https://doi.org/10.1249/ MSS.0b013e31817f1988

Kayser B, Verges S (2013) Cardiovascular and cerebrovascular responses to acute hypoxia following exposure to intermittent hypoxia in healthy humans. Obes Rev 14:579-592. https://doi. org/10.1113/jphysiol.2009.171553

Kong Z, Zang Y, Hu Y (2014) Normobaric hypoxia training causes more weight loss than normoxia training after a 4-week residential camp for obese young adults. Sleep Breath 18:591-597. https://doi.org/10.1152/ajpheart.00253.2009

Lim SS, Vos T, Flaxman AD et al (2012) A comparative risk assessment of burden of disease and injury attributable to 67 risk factors and risk factor clusters in 21 regions, 1990-2010: a systematic analysis for the Global Burden of Disease Study
2010. Lancet 380:2224-2260. https://doi.org/10.1016/S0140 $-6736(12) 61766-8$

Mackenzie R, Maxwell N, Castle P et al (2011) Acute hypoxia and exercise improve insulin sensitivity (SI2*) in individuals with type 2 diabetes. Diabetes Metab Res Rev 27:94-101. https://doi. org/10.1210/jc.2007-1734

Malfatto G, Ochoa JE, Parati G (2009) Effects of hypoxia on blood pressure regulation: interval hypoxic training as compared to obstructive sleep apnea-the other side of the coin? J Hypertens 27:1527-1532. https://doi.org/10.1097/HJH.0b013e3283300d6d

Manukhina EB, Downey HF, Mallet RT (2006) Role of nitric oxide in cardiovascular adaptation to intermittent hypoxia. Exp Biol Med (Maywood) 231:343-365

Milanović Z, Pantelić S, Trajković N et al (2013) Age-related decrease in physical activity and functional fitness among elderly men and women. Clin Interv Aging 8:549-556. https://doi.org/10.2147/ CIA.S44112

Morishima T, Kurihara T, Hamaoka T, Goto K (2013) Whole body, regional fat accumulation, and appetite-related hormonal response after hypoxic training. Clin Physiol Funct Imaging 34:90-97. https ://doi.org/10.1007/s00421-002-0606-4

Morishima T, Hasegawa Y, Sasaki H et al (2015) Effects of different periods of hypoxic training on glucose metabolism and insulin sensitivity. Clin Physiol Funct Imaging 35:104-109. https://doi. org/10.1111/cpf.12133

Naci H, Salcher-Konrad M, Dias S et al (2019) How does exercise treatment compare with antihypertensive medications? A network meta-analysis of 391 randomised controlled trials assessing exercise and medication effects on systolic blood pressure. $\mathrm{Br} \mathrm{J}$ Sports Med 53:859-869. https://doi.org/10.1136/bjsports-2018-099921

Nishiwaki M, Kawakami R, Saito K et al (2010) Vascular adaptations to hypobaric hypoxic training in postmenopausal women. J Physiol Sci 61:83-91. https://doi.org/10.1161/hypertensionaha .107 .101014

Parati G, Ochoa JE, Torlasco C et al (2015) Aging, high altitude, and blood pressure: a complex relationship. High Alt Med Biol 16:97109. https://doi.org/10.1089/ham.2015.0010

Pedralli ML, Marschner RA, Kollet DP et al (2020) Different exercise training modalities produce similar endothelial function improvements in individuals with prehypertension or hypertension: a randomized clinical trial exercise, endothelium and blood pressure. Sci Rep 10:7628. https://doi.org/10.1038/s41598-020-64365-x

Pramsohler S, Burtscher M, Faulhaber M et al (2017) Endurance training in normobaric hypoxia imposes less physical stress for geriatric rehabilitation. Front Physiol 8:683. https://doi.org/10.1016/j. cardiores.2006.09.003

Rapsomaniki E, Timmis A, George J et al (2014) Blood pressure and incidence of twelve cardiovascular diseases: lifetime risks, healthy life-years lost, and age-specific associations in 1.25 million people. Lancet 383:1899-1911. https://doi.org/10.1016/S0140 $-6736(14) 60685-1$

Schobersberger W, Schmid P, Lechleitner M et al (2003) Austrian Moderate Altitude Study 2000 (AMAS 2000). The effects of moderate altitude $(1700 \mathrm{~m})$ on cardiovascular and metabolic variables in patients with metabolic syndrome. Eur J Appl Physiol 88:506514. https://doi.org/10.1007/s00421-002-0736-8

Serebrovskaya TV, Xi L (2016) Intermittent hypoxia training as nonpharmacologic therapy for cardiovascular diseases: practical analysis on methods and equipment. Exp Biol Med (Maywood) 241:1708-1723. https://doi.org/10.1177/1535370216657614

Serebrovskaya TV, Manukhina EB, Smith ML et al (2008) Intermittent hypoxia: cause of or therapy for systemic hypertension? Exp Biol Med (Maywood) 233:627-650. https://doi.org/10.1089/ ham.2006.7.72 
Somers VK, Conway J, Johnston J, Sleight P (1991) Effects of endurance training on baroreflex sensitivity and blood pressure in borderline hypertension. Lancet 337:1363-1368

Stewart KJ, Bacher AC, Turner KL et al (2005) Effect of exercise on blood pressure in older persons: a randomized controlled trial. JAMA Internal Med 165:756-762. https://doi.org/10.1001/archi nte.165.7.756

Vogt M, Puntschart A, Geiser J et al (2001) Molecular adaptations in human skeletal muscle to endurance training under simulated hypoxic conditions. J Appl Physiol 91:173-182. https://doi. org/10.1152/jappl.2001.91.1.173

Wang J-S, Chen L-Y, Fu L-L et al (2007) Effects of moderate and severe intermittent hypoxia on vascular endothelial function and haemodynamic control in sedentary men. Eur J Appl Physiol 100:127-135. https://doi.org/10.1161/01.ATV.19.6.1559
Whelton PK, Carey RM, Aronow WS et al (2017) 2017 ACC/AHA/ AAPA/ABC/ACPM/AGS/APhA/ASH/ASPC/NMA/PCNA guideline for the prevention, detection, evaluation, and management of high blood pressure in adults: a report of the American College of Cardiology/American Heart Association Task Force on Clinical Practice Guidelines. J Am Coll Cardiol. https://doi.org/10.1016/j. jacc.2017.11.006

Wiesner S, Haufe S, Engeli S et al (2009) Influences of normobaric hypoxia training on physical fitness and metabolic risk markers in overweight to obese subjects. Obesity (Silver Spring) 18:116-120. https://doi.org/10.1038/oby.2009.193

Publisher's Note Springer Nature remains neutral with regard to jurisdictional claims in published maps and institutional affiliations. 